\title{
Bone Marrow Transplantation Nephropathy
}

National Cancer Institute

\section{Source}

National Cancer Institute. Bone Marrow Transplantation Nephropathy. NCI Thesaurus.

Code C5110.

Kidney dysfunction resulting from complications of bone marrow transplantation. 